\title{
Roles of poly (ADP-ribose) polymerase (PARP1) cleavage in the ovaries of fetal, neonatal, and adult pigs
}

\author{
Quanwei Wei, Wei Ding ${ }^{1}$ and Fangxiong Shi \\ Laboratory of Animal Reproduction, College of Animal Science and Technology, Nanjing Agricultural University, \\ Nanjing 210095, China and ${ }^{1}$ Department of Animal Husbandry and Veterinary Medicine, Jiangsu Polytechnic \\ College of Agriculture and Forestry, Jurong 212400, China
}

Correspondence should be addressed to F Shi; Email: fxshi@njau.edu.cn

\begin{abstract}
Poly(ADP-ribosylation), which occurs rapidly in cells following DNA damage and is regulated by poly (ADP-ribose) polymerase 1 (PARP1), is a post-translational modification of proteins playing a crucial role in many processes, including DNA repair and cell death. Although PARP1 has recently been implicated in a variety of physiological and pathological processes, its role in the process of follicular development and atresia is not yet completely defined. This study was designed to investigate the cellular expression pattern and immunolocalization of PARP1, cleaved PARP1, caspase 3, and cleaved caspase 3 in fetal, neonatal, and adult porcine ovaries. Our results showed that in fetal and neonatal pigs, PARP1 cleavage is involved in the process of oocyte nest breakdown, primordial follicle formation, and transition to primary follicles. The results of immunohistochemistry indicated that PARP1 cleavage was involved in the process of follicular development and atresia, which was in accordance with our previous study; however, it was noted that cleaved caspase 3 was mainly localized in and around the nucleus of apoptotic granulosa cells (GCs), whereas cleaved PARP1 was mainly localized in the nucleus of the apoptotic GCs. RIA data showed increased serum progesterone and estradiol concentrations with age after birth. Collectively, our findings suggest that the PARP1 signaling pathway is involved in oocyte nest breakdown and primordial follicle formation in fetal and neonatal porcine ovaries, but is different from follicular atresia in adult porcine ovaries that involves cellular apoptosis.

Reproduction (2013) 146 593-602
\end{abstract}

\section{Introduction}

Female fertility is affected by a variety of environmental, behavioral, and genetic factors that can alter folliculogenesis at various levels (Joffe 2003). These factors include a wide variety of endogenous and exogenous genotoxic agents that may lead to DNA damage and cell death. It was suggested that a germ cell can be lost to death processes before or after it is surrounded by somatic cells so as to form a follicle (Tingen et al. 2009a). The death of germ cells preceding follicle formation is termed attrition, whereas the degenerative process of follicle death once germ cells are surrounded by somatic cells or granulosa cells (GCs) is termed follicular atresia (Hsueh et al. 1994, Tingen et al. 2009b). In the mammalian ovary, each germ cell is either selected to grow and ovulate or be lost to an atretic pathway. In our previous work, we have reported that female reproductive capacity requires a balance between these two fates (Wei \& Shi 2013).

Poly (ADP-ribose) polymerase 1 (PARP1) is a $116 \mathrm{kDa}$ protein, the founding member of the PARP family which contains as many as 18 distinct proteins in humans, is a nuclear, and $\mathrm{NAD}^{+}$-dependent enzyme present in eukaryotes (Virag \& Szabo 2002, Kim et al. 2005). PARP1 can specifically bind and repair DNA-strand breaks generated by several genotoxic agents (Yelamos et al. 2011); apart from this, it is also implicated in the regulation of a wide range of important cellular processes including transcriptional regulation, chromatin modification, cellular homeostasis, and cell proliferation and death (Bernstein et al. 2002, Hassa 2009, Lu et al. 2011).

PARP1 protein has a modular structure composed of at least six domains, four of which have well-defined functions (Langelier et al. 2010, Megnin-Chanet et al. 2010). It is believed that these PARP1 domains may play roles in a variety of pathological processes leading to cell death, and which are mediated through PARP1 cleavage by suicide proteases including caspase 3 . Cleavage of PARP1 then results in exposed structural domains that mediate specific forms of cell death (Kim et al. 2005, Luo \& Kraus 2012). PARP1 cleavage produces an $89 \mathrm{kDa}$ C-terminal fragment, which always contains the catalytic domain; and a $24 \mathrm{kDa} N$-terminal fragment (Nguewa et al. 2003, Agarwal et al. 2009). The 89 kDa C-terminus 
fragment retains basal enzymatic activity due to the maintenance of the catalytic domain (Kim et al. 2005); however, PARP1 becomes inactive after cleavage, as it fails to recognize DNA damage. As a result, NAD ${ }^{+}$ consumption is prevented.

In the porcine ovary, oocyte nest breakdown and primordial follicle formation take place $\sim 90$ days post coitum (90 dpc), and the major stage of transition from primordial follicles into primary follicles was between $90 \mathrm{dpc}$ to 1 day post partum (1 dpp), according to our previous study (Ding et al. 2010). Ovarian follicle development is characterized by the processes of GC proliferation, differentiation, and apoptosis. Thereby, GCs are crucial for directing normal follicular development and atresia (Fan et al. 2008, Wei \& Shi 2013); however, the exact signaling pathway is still unclear.

Our previous study demonstrated that cleavage of PARP1 by caspase 3 is strongly implicated in porcine follicular atresia (Wei \& Shi 2013), and there is evidence that PARP1 may take part in folliculogenesis (Qian et al. 2010); however, the biological mechanisms responsible for follicular atresia are not as well studied as those responsible for earlier germ cell attrition. The aim of this study is to expand our knowledge of PARP1 cleavage signaling pathway during the process of follicular development and atresia in fetal, neonatal, and adult pigs.

\section{Materials and methods}

\section{Ethics statement}

Animal procedures were conducted in accordance with the guidelines of the Institutional Animal Care and Use Committee of Nanjing Agricultural University. All pigs were killed in a state of unconsciousness, and then the ovaries were removed.

\section{Reagents}

Expression patterns and location of PARP1, PARP1 antibody (cleaved p25), caspase 3, and cleaved-caspase 3 (Asp175) were analyzed by immunohistochemistry (IHC) and immunoblotting. PARP1 antibody H-250 was purchased from Santa Cruz. PARP1 antibody (cleaved p25) is a reagent originally manufactured by Abgent (San Diego, CA, USA). The cleavedcaspase 3 (Asp175) antibody was purchased from Cell Signaling (Boston, MA, USA). Caspase 3 antibody and immunohistochemical kits (SABC method) were purchased from Boshide (Wuhan, China). 3,3-Diaminobenzidine tetrachloride (DAB) was purchased from Sigma-Aldrich. Estradiol$17 \beta\left(E_{2}\right)$ and progesterone $\left(\mathrm{P}_{4}\right)$ RIA kits were purchased from Beijing North Biotechnology Institute, China. All other chemicals were purchased reagent grade.

\section{Animals and conditions}

Sexually mature gilts, all at 9-10 months of age and with body weights of 100-120 kg, were obtained from Jiangpu Farms of Nanjing Agricultural University (Nanjing, China). The animals were kept indoors and provided with commercial foods three times per day and tap water ad libitum. After exhibiting at least two estrous cycles of normal duration, gilts were observed for estrous behavior twice daily and mated to fertile boars at the onset of estrus and 12 and $24 \mathrm{~h}$ later. The gestation day was estimated from the first mating day.

\section{Collection of fetal, neonatal, and adult porcine ovaries}

Fetal porcine ovaries from pregnant gilts were obtained by hysterotomy performed under anesthesia with lidocaine on day $90 \mathrm{dpc}$. Neonatal porcine ovaries from neonatal pups were obtained by ovariectomy performed under anesthesia with lidocaine on $1 \mathrm{dpp}$. Adult porcine ovaries were obtained from mature sows (150 dpp) at Nanjing Tianhuan Company (Jiangsu province, China), a local slaughterhouse. Before slaughter, pigs are first rendered unconscious using the following means: stunning using electric current applied with electrodes and then hoisted on a rail, after which they are exsanguinated via the carotid artery. After slaughter, the ovaries were removed and carried to the laboratory as soon as possible in physiological saline at $30-35^{\circ} \mathrm{C}$. One ovary was fixed in $4 \%$ paraformaldehyde at room temperature for $36 \mathrm{~h}$ and then kept in $70 \%$ alcohol for IHC; the contralateral ovary was frozen and kept refrigerated at $-80^{\circ} \mathrm{C}$.

\section{IHC of fetal, neonatal, and adult porcine ovaries}

To examine the locations of PARP1, cleaved PARP 1, caspase 3, and cleaved caspase 3 in porcine ovaries, ovarian sections from fetal, neonatal, and adult pigs were prepared. Immunohistochemical staining was performed with the SABC method using MABs to PARP 1 (diluted 1:200), cleaved PARP 1 (1:300), caspase 3 (1:150), or cleaved caspase 3 (1:400). A few sections were picked randomly from the serial sections, and mounted on slides coated with 3-aminopropyl-triethoxysilane and dried for $24 \mathrm{~h}$ at $37^{\circ} \mathrm{C}$. The antibodies were diluted in PBS containing $1 \%$ (W/V) BSA, and the sections were incubated overnight at $4{ }^{\circ} \mathrm{C}$ with primary antibodies. The specific protein immunoreactivity was visualized with $0.05 \%$ DAB in $10 \mathrm{mM}$ PBS containing $0.01 \%$ (V/V) $\mathrm{H}_{2} \mathrm{O}_{2}$ for $2 \mathrm{~min}$, and counterstained with hematoxylin. The negative control was normal rabbit serum instead of primary antibody, and relative levels of immunostaining were evaluated according to our previous study (Ding et al. 2010). The images were captured under the microscope. To assign the intensities of staining for PARP 1, cleaved PARP 1, caspase 3, and cleaved caspase 3, three independent observers blinded to the experimental design were asked to examine the pictures, using a method previously described (Wei et al. 2012): - , no staining detected; + , weak; ++ , moderate; and +++ , strong staining. Relative levels of immunostaining were evaluated and repeated at least four times.

\section{Classification of porcine ovarian follicles}

In order to study the histological changes as well as expression and localization of PARP1, cleaved PARP1, caspase 3, and cleaved caspase 3 by IHC during the process of porcine follicular development and atresia, each follicle was classified histologically as an unassembled oocyte (remaining in 
Table 1 Classification criteria of porcine ovarian follicles ${ }^{\mathrm{a}}$.

\begin{tabular}{ll}
\hline Follicle & Morphologic characteristics of cells \\
\hline Unassembled oocytes & Unassembled oocytes are surrounded by a single layer of squamous stromal tissue (remaining in oocyte nests) \\
Primordial follicles & Oocyte partially or completely encapsulated by squamous pregranulosa cells \\
Primary follicles & Granulosa cells (with single layer) show enlargement \\
Preantral follicles & Oocyte encapsulated by more than two layers of granulosa cells; no antrum formation \\
Antral follicles & Oocyte encapsulated by more than two layers of granulosa cells with antrum \\
Atretic follicles & $\begin{array}{l}\text { Follicles with apoptotic and shedding granulosa cells in the follicular antrum, and apoptosis of granulosa cells } \\
\text { accompanied by the presence of nuclei with condensed chromatin stained with hematoxylin }\end{array}$ \\
\hline
\end{tabular}

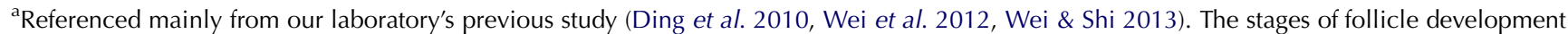
(unassembled, primordial, primary, preantral, antral, and atretic follicles) were divided based on the classification criteria provided in this table.

oocyte nests), or as a primordial, primary, preantral, antral, or atretic follicle. According to our previous study (Ding et al. 2010, Wei et al. 2012, Wei \& Shi 2013), porcine ovarian follicles were classified based on the classification criteria of Table 1.

\section{RIA for $E_{2}$ and $P_{4}$ concentrations in the serum of neonatal, young, and adult pigs}

Blood was collected from pigs of different ages and centrifuged at $5000 \boldsymbol{g}$ for $10 \mathrm{~min}$ to separate the serum. The serum was used to quantify $E_{2}$ and $P_{4}$ using $E_{2}$ and $P_{4}$ RIA kits, respectively, in the General Hospital of the Nanjing Military Command.

\section{SDS-PAGE and western blot analysis}

Proteins from porcine ovaries were prepared for western blot analysis in RIPA lysis buffer (Beyotime, Nantong, China) containing $10 \mathrm{mM}$ phenylmethylsulfonyl fluoride (Beyotime) using a Dounce homogenizer. After homogenization, samples were incubated for $30 \mathrm{~min}$ on ice, and centrifuged at $10000 \mathrm{~g}$ for $10 \mathrm{~min}$ at $4{ }^{\circ} \mathrm{C}$. The supernatant was separated and the protein concentration was determined using a BCA Protein Assay Kit (Beyotime). In each experiment, equal amounts of sample lysate (15-40 $\mu \mathrm{g})$ were separated by $10 \%(\mathrm{w} / \mathrm{v})$ SDSPAGE under standard reducing conditions with precision protein molecular weight markers (Fermentas, Shanghai, China), and then electrotransferred onto PVDF membranes (Millipore, Bedford, MA, USA). Membranes were blocked with $2 \%$ BSA in TBST buffer (20 mM Tris-buffered saline, 0.05\% Tween 20, pH 7.5) for $2 \mathrm{~h}$ at room temperature and incubated for $18 \mathrm{~h}$ at $4{ }^{\circ} \mathrm{C}$ with diluted antibodies specific to PARP1 (diluted 1:1000), cleaved PARP1 (1:2500), and $\boldsymbol{\alpha}$-tubulin (1:5000). Subsequently, the membranes were washed with TBST buffer and incubated with HRP-linked secondary goat anti-rabbit IgG antibody (diluted at 1:2000) for $3 \mathrm{~h}$. Finally, the blots were washed four times and visualized with Super Signal West Pico Chemiluminescent Subs Kits (Pierce Biotechnology, Shanghai, China).

\section{Statistical analysis}

All results are expressed as mean \pm S.E.M. All statistical analyses were performed using GraphPad Prism 5 (GraphPad Software, Inc., San Diego, CA, USA). Students t-test was used to compare two groups, and a one-way ANOVA was used for more than two groups, with Tukey's range test applied for multiple comparisons. Statistical significant difference was considered to be $P<0.05$.

\section{Results}

\section{Neonatal, young, and adult pigs showed increased $P_{4}$ and $E_{2}$ concentrations with time after birth}

During the process of oophorectomy, the serum of neonatal and young pigs (5, 35, 70, and $180 \mathrm{dpp})$ was also gathered.

The serum was used to quantify $\mathrm{E}_{2}$ and $\mathrm{P}_{4}$ levels using $E_{2}$ and $P_{4}$ RIA kits. The concentration of $E_{2}$ and $P_{4}$ in the serum obtained from pigs of different ages is shown in Fig. 1. The data showed that there were increased $P_{4}$
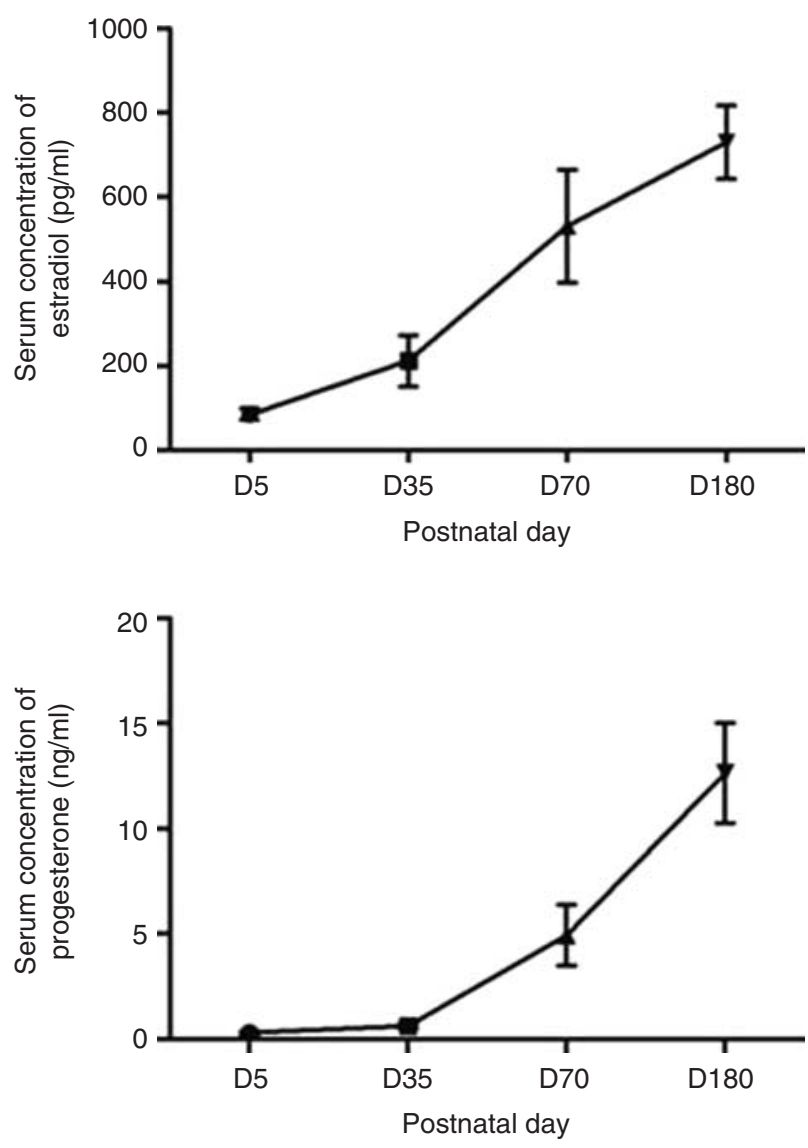

Figure 1 Serum concentrations of progesterone $\left(P_{4}\right)$ and estradiol $\left(E_{2}\right)$ in pigs of different ages $(5,35,70$, and $180 \mathrm{dpp})$. Data represent the mean \pm s.E.M. from three duplicates. D5, D35, D70, and D180 stand for $5,35,70$, and $180 \mathrm{dpp}$ respectively. Significant differences by ANOVA and Tukey's post-hoc test, $n=3$. 
and $E_{2}$ concentrations after birth with age $(5,35,70$, and $180 \mathrm{dpp})$.

\section{The PARP1 cleavage signaling pathway exists in the unassembled oocytes (oocyte nests), but not in the primordial follicles of fetal and neonatal porcine ovaries}

As described above, according to our previous study, two key time points (90 and $1 \mathrm{dpp}$ ) were selected as fetal and neonatal period to study the role of the PARP1 cleavage signaling pathway during the process of oocyte nest breakdown and primordial follicle formation and development in fetal and neonatal porcine ovaries. We investigated the cellular localization patterns of PARP1, cleaved PARP1, caspase 3, and cleaved caspase 3 in the ovary of pig by IHC. The porcine ovarian follicles were classified based on the classification criteria in Table 1. In the porcine ovary, positive reaction was observed as brown staining (marked with red arrows). No specific staining was observed in the negative control sections (Fig. 2E1 and $\mathrm{E} 2$, Fig. $3 \mathrm{E} 1$ and E2). The results showed that the immunostaining pattern of PARP1, cleaved PARP1, caspase 3, and cleaved caspase 3 was varied among different follicle stages during the process of oocyte nest breakdown, primordial follicle formation, and transition to primary follicles. Relative expression levels of immunostaining of PARP1, cleaved PARP1 caspase 3, and cleaved caspase 3 in the ovaries are shown in Table 2.

Immunohistochemical results showed that PARP1 and caspase 3 were localized extensively to healthy oocytes of the oocyte nests and primordial follicles (Fig. 2A1, A2, C1 and C2, Fig. 3A1, A2, C1 and C2). The results also showed that oocyte nest breakdown was accompanied by caspase 3 activation (cleaved caspase 3) and PARP1 deactivation (cleaved PARP1) in the apoptotic oocytes (Fig. 2). In the nonapoptotic oocytes, there was almost no staining of cleaved caspase 3 and cleaved PARP1 (Fig. 2D1), while immunostaining of PARP1 and caspase 3 was clearly observed in the nucleus and cytoplasm of the healthy oocytes (Fig. 2D2). In pregranulosa cells, the staining of PARP1 and caspase 3 was weak, while that of the cleaved caspase 3 and cleaved PARP1 was almost invisible (Fig. 2E1, E2 and E3). Interestingly, Fig. 2C1, $\mathrm{C} 2$ and C3 clearly showed that cleaved PARP1 was mainly located in the nucleus; (however, the cleaved caspase 3 was located around the nucleus of the apoptotic oocytes; Table 2).

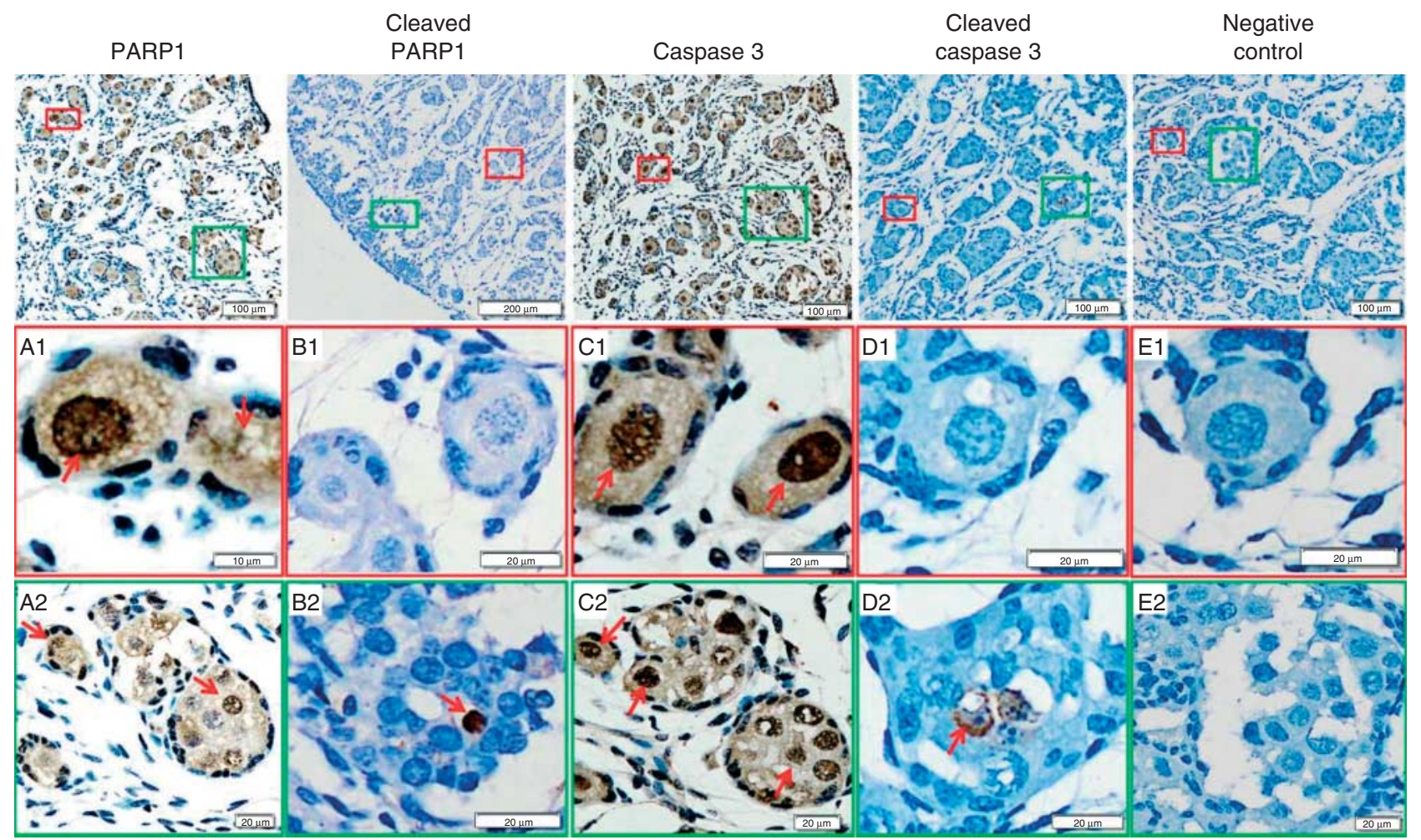

Figure 2 Immunostaining of PARP1 (A), cleaved PARP1 (B), caspase 3 (C), and cleaved caspase 3 (D) in the porcine ovary (90 dpc). The negative control (E) was normal rabbit serum (NRS) instead of primary antibody (photographs in differently colored individual square boxes marked above are enlarged below and marked A1, 2, B1, 2, C1, 2, D1, 2, E1, and 2). Staining of PARP1 (A), cleaved PARP1 (B), caspase 3 (C), and cleaved caspase 3 (D) was qualitatively different in oocytes at various stages of germ cell growth and development. 


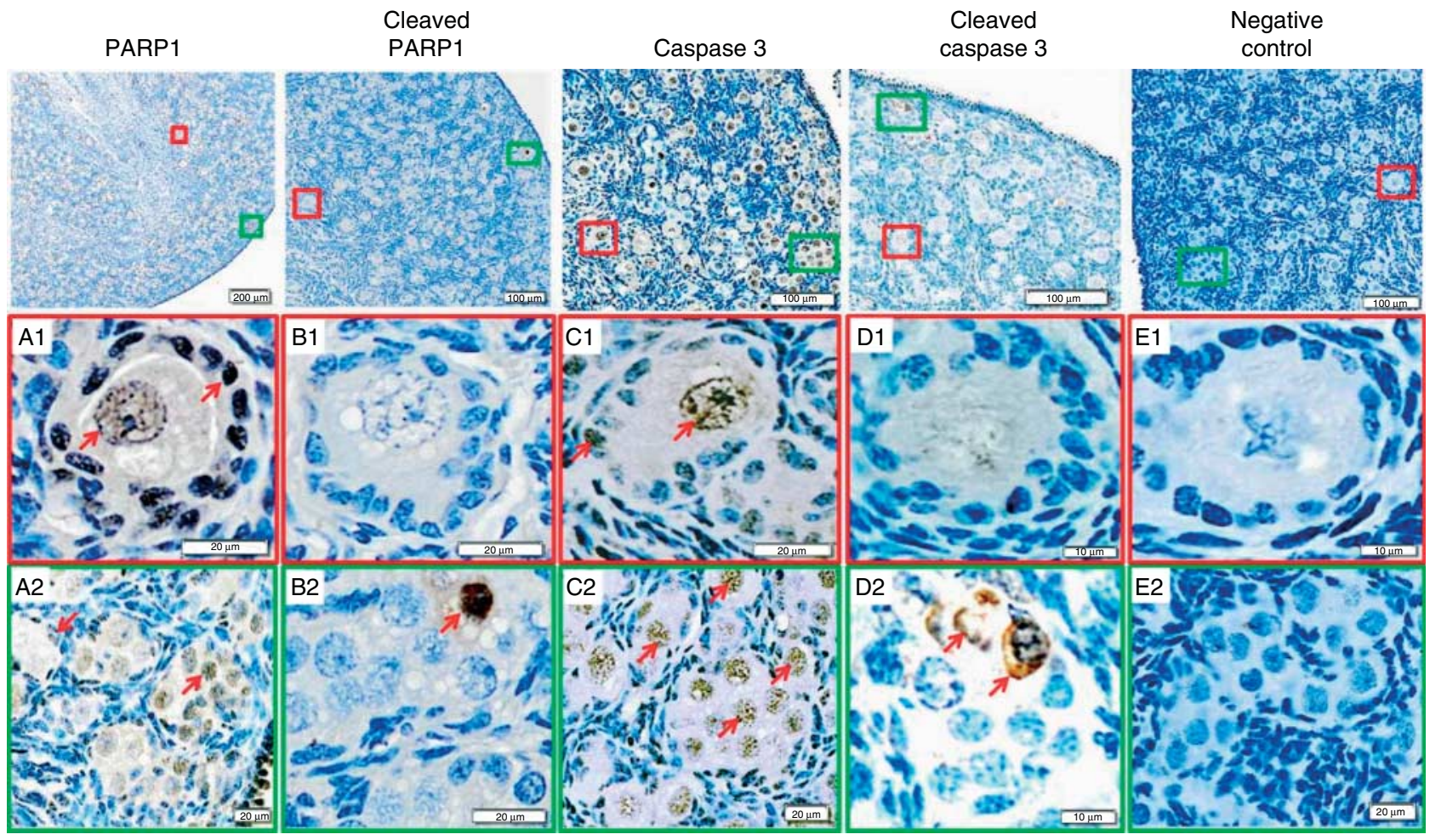

Figure 3 Immunostaining of PARP1 (A), cleaved PARP1 (B), caspase 3 (C), and cleaved caspase 3 (D) in the porcine ovary (1 dpp). The negative control (E) was normal rabbit serum (NRS) instead of primary antibody (photographs in differently colored individual square boxes marked above were enlarged below and marked A1, 2, B1, 2, C1, 2, D1, 2, E1, and 2). Staining of PARP1 (A), cleaved PARP1 (B), caspase 3 (C), and cleaved caspase 3 (D) was qualitatively different in the oocytes at various stages of germ cell growth and development.

\section{Degeneration of the porcine ovarian follicle wall and GC apoptosis is accompanied by caspase 3 activation and PARP1 cleavage}

Our previous study demonstrated that cleavage of PARP1 may serve a key role in controlling follicular atresia through degeneration of GC. In order to further study the characteristics of PARP1 cleavage and caspase 3 cleavage during the process of porcine ovarian follicle wall degeneration, two kinds of atretic follicles (follicles with both healthy GCs and shedding, apoptotic GCs (Fig. 4A1, B1, C1, D1 and E1), and follicles with almost all the GCs as shedding and apoptotic (Fig. 4A2, B2, C2, D2 and E2)) were examined using PARP1, cleaved PARP1 caspase 3, and cleaved caspase 3 antibodies by IHC (Table 3 ).

The apoptosis of GCs was accompanied by the presence of nuclei with condensed chromatin stained with

Table 2 Relative levels of immunostaining of PARP1, cleaved PARP1, caspase 3, and cleaved caspase 3 in the nonapoptotic cells of fetal and neonatal porcine ovaries.

\begin{tabular}{|c|c|c|c|c|c|}
\hline Protein & Age & Stages & Unassembled follicle & Primordial follicle & Primary follicle \\
\hline \multirow[t]{4}{*}{ PARP1 } & $90 \mathrm{dpc}$ & Oocyte & ++ & +++ & NA \\
\hline & & Granulosa cells & NA & + & NA \\
\hline & $1 \mathrm{dpp}$ & Oocyte & ++ & +++ & +++ \\
\hline & & Granulosa cells & NA & ++ & ++ \\
\hline \multirow[t]{4}{*}{ Cleaved PARP1 } & $90 \mathrm{dpc}$ & Oocyte & - & - & - \\
\hline & & Granulosa cells & NA & - & NA \\
\hline & $1 \mathrm{dpp}$ & Oocyte & - & - & - \\
\hline & & Granulosa cells & NA & - & - \\
\hline \multirow[t]{4}{*}{ Caspase 3} & $90 \mathrm{dpc}$ & Oocyte & + & ++ & NA \\
\hline & & Granulosa cells & NA & + & NA \\
\hline & $1 \mathrm{dpp}$ & Oocyte & +++ & +++ & +++ \\
\hline & & Granulosa cells & NA & ++ & ++ \\
\hline \multirow[t]{4}{*}{ Cleaved caspase 3} & $90 \mathrm{dpc}$ & Oocyte & - & - & - \\
\hline & & Granulosa cells & NA & - & NA \\
\hline & $1 \mathrm{dpp}$ & Oocyte & & & \\
\hline & & Granulosa cells & NA & - & - \\
\hline
\end{tabular}

- , No staining detected; + , weak; ++ , moderate; +++ , strong staining; NA, not available. 


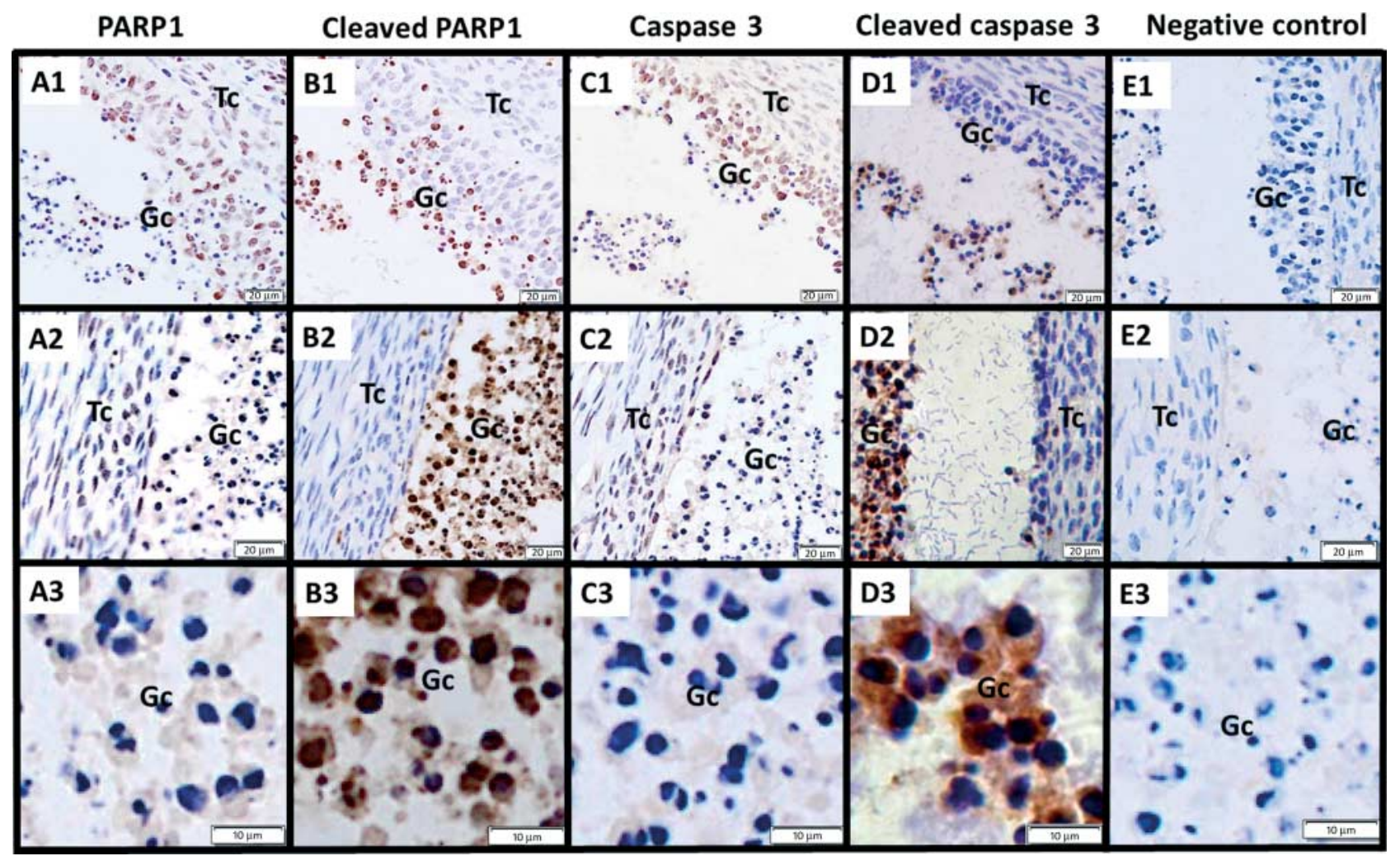

Figure 4 Immunostaining of PARP1 (A), cleaved PARP1 (B), caspase 3 (C), and cleaved caspase 3 (D) in granulosa cells during the process of follicular atresia (150 dpp). The negative control (E) was normal rabbit serum (NRS) instead of primary antibody. From the enlarged pictures (A3-E3), it was more clearly demonstrated that PARP- 1 and caspase- 3 could not be detected in the apoptotic GCs, however, cleaved PARP- 1 was mainly located in the nucleus of GCs, whereas cleaved caspase- 3 was in and around the nucleus. Atretic follicles are characterized by shedding and apoptotic granulosa cells in the follicular antrum, and the apoptosis of granulosa cells was accompanied by the presence of nuclei with condensed chromatin stained with hematoxylin.

hematoxylin. The results showed that the cleavage of PARP1 by cleaved caspase 3 was in apoptotic GCs, which is consistent with our previous findings (Wei \& Shi 2013). However, there was an interesting phenomenon-cleaved caspase 3 was mainly localized in and around the nucleus of apoptotic GCs, whereas cleaved PARP1 was mainly localized to the nucleus (Fig. 4A2, B2, C2, D2 and E2).

\section{Expression patterns of PARP1 and cleaved PARP1 in fetal, neonatal, and adult porcine ovaries}

To provide more evidence elucidating the role of PARP1 cleavage during the process of oocyte nest breakdown, primordial follicle formation, and development in fetal and neonatal porcine ovaries, the expression patterns of PARP1 and cleaved PARP1 in porcine ovarian extracts from fetal neonatal and adult porcine ovaries were analyzed by western blotting.

The data showed that PARP1 cleavage produces an $89 \mathrm{kDa}$ C-terminal fragment, and a $24 \mathrm{kDa} \mathrm{N}$-terminal fragment in fetal (90 dpc), neonatal (1 dpp), and adult (150 dpp) porcine ovaries (Fig. 5). Thus, the results of western blotting indicated that porcine ovarian development was accompanied by PARP1 cleavage.

\section{Involvement of the PARP1 cleavage signaling pathway during the process of follicular development and atresia in adult porcine ovaries}

To study the role of PARP1 cleavage during the process of follicular development and atresia, we investigated the characteristics of localization of PARP1, cleaved PARP1 caspase 3, and cleaved caspase 3 proteins in the adult porcine ovary by IHC. In the ovary, a positive reaction was observed as brown staining. No specific staining was observed in the negative control sections. The porcine ovarian follicles were classified based on the classification criteria in Table 1. The results showed that the immunostaining patterns of PARP1, cleaved

Table 3 Relative levels of immunostaining of PARP1, cleaved PARP1, and caspase 3 in the granulosa cells (GCs) from porcine ovaries.

\begin{tabular}{lcc}
\hline Status & Healthy & Apoptotic \\
\hline PARP1 & +++ & - \\
Cleaved PARP1 & - & +++ \\
Caspase 3 & +++ & - \\
Cleaved caspase 3 & - & +++ \\
\hline
\end{tabular}

- , No staining detected; + , weak; ++ , moderate; +++ , strong staining; NA, not available. 
A
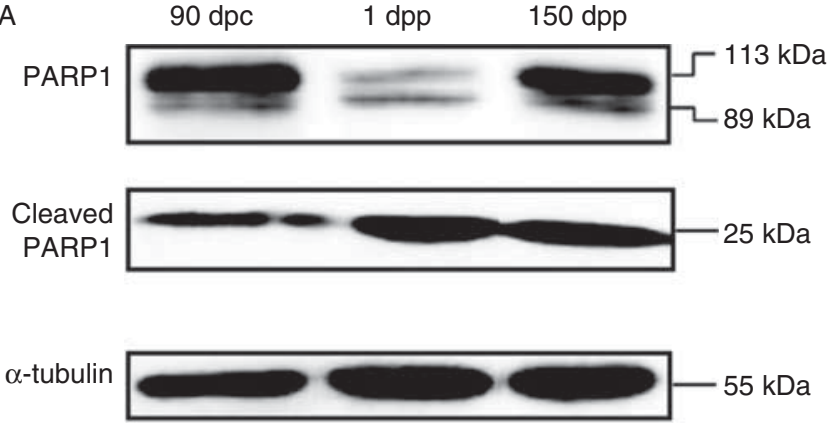

B

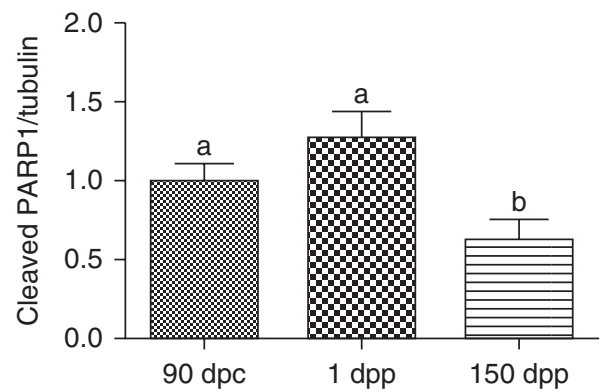

Figure 5 (A) Analysis of PARP1, cleaved PARP1, and tubulin expression in fetal (90 dpc), neonatal (1 dpp), and adult (150 dpp) porcine ovaries. dpc, days post coitum; dpp, day post partum. $T$ Significant differences by ANOVA and Tukey's post-hoc test, $n=3$. (B) Bars with different letters are significantly different from one another $(P<0.05)$.

PARP1 caspase 3, and cleaved caspase 3 were varied between healthy and atretic follicles. Relative levels of immunostaining of PARP1, and cleaved PARP1 and caspase 3 in the GCs of porcine ovaries are shown in Table 4.

Immunohistochemical results showed that PARP1 and caspase 3 were extensively localized to healthy GCs and oocytes of primary follicles, secondary follicles, early antral follicles, and antral follicles in the porcine ovary (Fig. 6). In the atretic follicles, there was almost no staining of PARP1 (Fig. 6A5) or caspase 3 (Fig. 6C5) in the apoptotic GCs, while immunostaining of cleaved caspase 3 (Fig. 6D5) and cleaved PARP1 (Fig. 6B5) was clearly observed.

\section{Discussion}

In this study, we make a case for cleavage of PARP1 being strongly implicated in porcine follicular development and atresia of fetal, neonatal, and adult porcine ovaries. We demonstrated that PARP1 cleavage might play an important role during the process of follicular development and atresia of fetal, neonatal, and adult porcine ovaries using IHC and western blotting. The RIA data showed increased $\mathrm{P}_{4}$ and $\mathrm{E}_{2}$ concentrations with age after birth (5, 35, 70, and $180 \mathrm{dpp})$.
We provided evidences that the PARP1 cleavage signaling pathway exists in the unassembled oocytes (oocyte nests) during the process of oocyte nest breakdown and primordial follicle formation. However, according to our previous findings, the mechanisms involving PARP1 cleavage and controlling follicle atresia were in GCs but not in oocytes. Therefore, we concluded that PARP1 cleavage in oocytes was involved in the process of oocyte nest breakdown of porcine ovaries. Thus, the mechanisms underlying oocyte nest breakdown may be different from those of follicular atresia. The IHC results showed that cleaved caspase 3 was mainly localized in and around the nucleus of apoptotic GCs, whereas cleaved PARP1 was mainly localized in the nucleus; this indicated that the cleavage of PARP1 occurred in the nucleus after activation of caspase 3 in the cytoplasm during the process of follicular atresia. However, these findings need to be corroborated by more evidence.

DNA strand breaks can lead to PARP1 activation, and the activation of PARP1 may be an attempt to repair the DNA damage caused by nuclease activation in the dying cell (Spina-Purrello et al. 2008, Tanori et al. 2008). However, this attempt to repair damage proves futile as PARP1 is cleaved by cleaved caspase 3 into a catalytic fragment of an $89 \mathrm{kDa}$ and DNA binding unit of $24 \mathrm{kDa}$ (D'Amours et al. 1999, Agarwal et al. 2009). Therefore, this cleaved PARP1 could serve as a biochemical marker of caspase-dependent apoptosis.

During the process of cellular apoptosis, PARP1 activation and cleavage play active but complex roles (Boulares et al. 1999, Chaitanya et al. 2010). Although PARP1 cleavage is considered to be a prominent marker of apoptosis, it is worth recalling that apoptosis cannot be assessed exclusively by evaluating PARP1 proteolysis. PARP cleavage is an important step in the apoptotic process, but is only one of many. Although cleavage of PARP1 by cleaved caspase 3 at the DEVD site is a universal phenomenon during the process of cell apoptosis, the significance of this cleavage in vivo is largely unknown (Petrilli et al. 2004). Some studies showed that PARP1 cleavage might be an attempt to inactivate its capacity to repair DNA so as to preserve energy pools (Herceg \& Wang 2001). There are findings that suggest that the $24 \mathrm{kDa}$-terminal PARP1 fragment irreversibly binds to DNA in order to prevent DNA repair

Table 4 Relative levels of immunostaining of PARP1, and cleaved PARP1, and caspase 3 in the granulosa cells (GCs) from porcine ovaries.

\begin{tabular}{lcc}
\hline Status & Healthy & Apoptotic \\
\hline PARP1 & +++ & - \\
Cleaved PARP1 & - & +++ \\
Caspase 3 & +++ & - \\
Cleaved caspase 3 & - & +++ \\
\hline
\end{tabular}

- , No staining detected; + , weak; ++ , moderate; +++ , strong staining; NA, not available. 


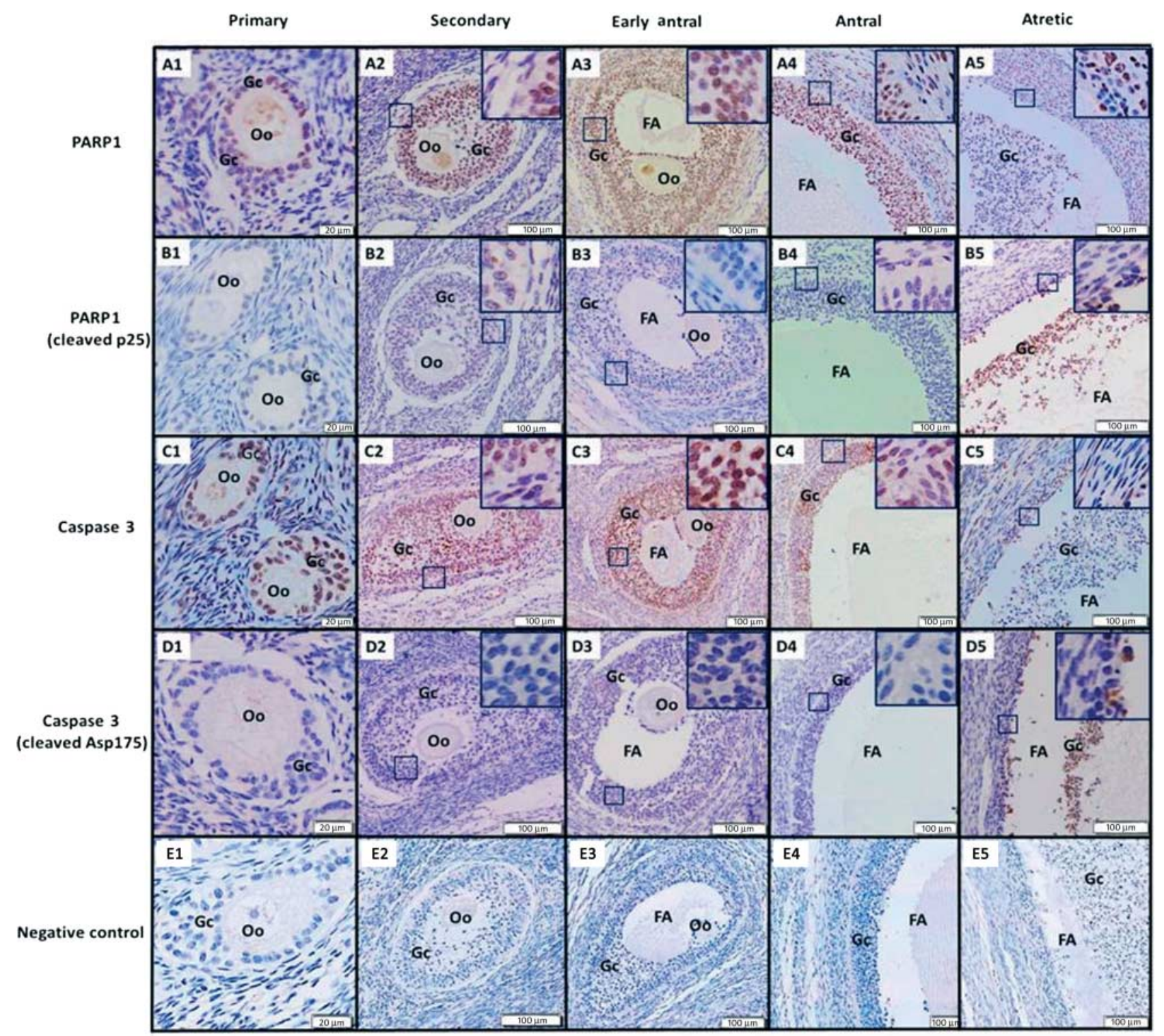

Figure 6 Immunostaining of PARP1 (A), cleaved PARP1 (B), caspase 3 (C), and cleaved caspase 3 (D) in the adult porcine ovary during follicular development and atresia (150 dpp). The negative control (E) was normal rabbit serum (NRS) instead of primary antibody (photographs in differently colored individual square boxes marked above were enlarged below and marked A1, 2, B1, 2, C1, 2, D1, 2, E1, and 2). Staining of PARP1 (A), cleaved PARP1 (B), caspase 3 (C), and cleaved caspase 3 (D) was qualitatively different in the oocytes at various stages of germ cell growth and development. Porcine ovarian follicles were classified based on the classification criteria in Table 1.

(Smulson et al. 1998, Petrilli et al. 2004). Thus, these findings indicate that the cleavage of PARP1 is an important regulatory event in cellular functions, but the mechanisms involved require clarification. In addition to apoptosis, necrosis and autophagy may play significant roles in cell death (Edinger \& Thompson 2004). What's more, evidences indicated that there was complex interplay between apoptosis, necrosis, and autophagy (Walsh \& Edinger 2010, Chaabane et al. 2013). It was reported that mTOR signaling, which may play key roles in autophagy, can be modulated by PARP1 (Munoz-Gamez et al. 2009, Ethier et al. 2012). Thus, a better understanding of the signaling pathway involved in cell death needs future studies to dissect the interplay among death, apoptosis, and necrosis in cell death and survival more thoroughly.

Our previous study demonstrated that oocyte nest breakdown and primordial follicle formation take place at $\sim 90 \mathrm{dpc}$, and the transition from primordial follicles to primary follicles was primarily between $90 \mathrm{dpc}$ and $1 \mathrm{dpp}$ in the porcine ovary (Ding et al. 2010); however, the mechanisms controlling these processes remain unclear. Follicular development and atresia are complex processes that are governed by a series of endocrine, 
paracrine, and autocrine signals (Matsuda et al. 2012). $E_{2}$ and $\mathrm{P}_{4}$ are two of the main steroid hormones that play essential roles during follicular development. The fate of follicles is then determined by the balance between/ among these factors, and it is believed that $E_{2}$ and $P_{4}$ play vital roles during the process of folliculogenesis (Kezele \& Skinner 2003, Wen et al. 2010). Our findings may provide further insight into understanding these processes.

\section{Conclusions}

In conclusion, our data suggest that the cleavage of PARP1 by activated (cleaved) caspase 3 plays an important role during the process of oocyte nest breakdown and primordial follicle formation, as well as in follicular development and atresia. We believe that these findings will prove to be helpful in investigating the mechanisms controlling follicular development and atresia.

\section{Declaration of interest}

The authors declare that there is no conflict of interest that could be perceived as prejudicing the impartiality of the research reported.

\section{Funding}

This study was supported in part by the National Nature Science Foundation of China (no. 31172206), Nature Science Foundation of Jiangsu Province, China (no. BK 2011499), and a Grant-in-Aid for Innovative Training of Doctoral Students in Jiangsu Province, China (no. CXZZ12-0294).

\section{Acknowledgements}

We thank Jiangpu Farms of Nanjing in China for supplying experimental animals. We also express our gratitude to Dr Reinhold J Hutz of the Department of Biological Sciences, University of Wisconsin-Milwaukee, USA, for reading the original manuscript and offering valuable suggestions.

\section{References}

Agarwal A, Mahfouz RZ, Sharma RK, Sarkar O, Mangrola D \& Mathur PP 2009 Potential biological role of poly (ADP-ribose) polymerase (PARP) in male gametes. Reproductive Biology and Endocrinology 7143 (doi:10.1186/1477-7827-7-143)

Bernstein C, Bernstein H, Payne CM \& Garewal H 2002 DNA repair/ pro-apoptotic dual-role proteins in five major DNA repair pathways: fail-safe protection against carcinogenesis. Mutation Research 511 145-178. (doi:10.1016/S1383-5742(02)00009-1)

Boulares AH, Yakovlev AG, Ivanova V, Stoica BA, Wang G, lyer S \& Smulson M 1999 Role of poly(ADP-ribose) polymerase (PARP) cleavage in apoptosis. Caspase 3-resistant PARP mutant increases rates of apoptosis in transfected cells. Journal of Biological Chemistry 274 22932-22940. (doi:10.1074/jbc.274.33.22932)
Chaabane W, User SD, El-Gazzah M, Jaksik R, Sajjadi E, RzeszowskaWolny J \& Los MJ 2013 Autophagy, apoptosis, mitoptosis and necrosis: interdependence between those pathways and effects on cancer. Archivum Immunologiae et Therapiae Experimentalis $61 \quad 43-58$. (doi:10.1007/s00005-012-0205-y)

Chaitanya GV, Steven AJ \& Babu PP 2010 PARP-1 cleavage fragments: signatures of cell-death proteases in neurodegeneration. Cell Communication and Signaling 8 31. (doi:10.1186/1478-811X-8-31)

D'Amours D, Desnoyers S, D'Silva I \& Poirier GG 1999 Poly(ADP-ribosyl)ation reactions in the regulation of nuclear functions. Biochemical Journal 342 249-268. (doi:10.1042/0264-6021:3420249)

Ding W, Wang W, Zhou B, Zhang W, Huang P, Shi F \& Taya K 2010 Formation of primordial follicles and immunolocalization of PTEN, PKB and FOXO3A proteins in the ovaries of fetal and neonatal pigs. Journal of Reproduction and Development 56 162-168. (doi:10.1262/ jrd.09-094H)

Edinger AL \& Thompson CB 2004 Death by design: apoptosis, necrosis and autophagy. Current Opinion in Cell Biology 16 663-669. (doi:10.1016/j. ceb.2004.09.011)

Ethier C, Tardif M, Arul L \& Poirier GG 2012 PARP-1 modulation of mTOR signaling in response to a DNA alkylating agent. PLOS ONE 7 e47978. (doi:10.1371/journal.pone.0047978)

Fan HY, Shimada M, Liu Z, Cahill N, Noma N, Wu Y, Gossen J \& Richards JS 2008 Selective expression of KrasG12D in granulosa cells of the mouse ovary causes defects in follicle development and ovulation. Development 135 2127-2137. (doi:10.1242/dev.020560)

Hassa PO 2009 The molecular "Jekyll and Hyde" duality of PARP1 in cell death and cell survival. Frontiers in Bioscience 14 72-111. (doi:10.2741/3232)

Herceg Z \& Wang ZQ 2001 Functions of poly(ADP-ribose) polymerase (PARP) in DNA repair, genomic integrity and cell death. Mutation Research 477 97-110. (doi:10.1016/S0027-5107(01)00111-7)

Hsueh AJ, Billig H \& Tsafriri A 1994 Ovarian follicle atresia: a hormonally controlled apoptotic process. Endocrine Reviews 15 707-724.

Joffe M 2003 Infertility and environmental pollutants. British Medical Bulletin 68 47-70. (doi:10.1093/bmb/ldg025)

Kezele P \& Skinner MK 2003 Regulation of ovarian primordial follicle assembly and development by estrogen and progesterone: endocrine model of follicle assembly. Endocrinology 144 3329-3337. (doi:10.1210/ en.2002-0131)

Kim MY, Zhang T \& Kraus WL 2005 Poly(ADP-ribosyl)ation by PARP-1: 'PAR-laying' $\mathrm{NAD}^{+}$into a nuclear signal. Genes and Development 19 1951-1967. (doi:10.1101/gad.1331805)

Langelier MF, Ruhl DD, Planck JL, Kraus WL \& Pascal JM 2010 The Zn3 domain of human poly(ADP-ribose) polymerase-1 (PARP-1) functions in both DNA-dependent poly(ADP-ribose) synthesis activity and chromatin compaction. Journal of Biological Chemistry 285 18877-18887. (doi:10.1074/jbc.M110.105668)

Lu H, Wang X, Li T, Urvalek AM, Yu L, Li J, Zhu J, Lin Q, Peng X \& Zhao J 2011 Identification of poly (ADP-ribose) polymerase-1 (PARP-1) as a novel Kruppel-like factor 8-interacting and -regulating protein. Journal of Biological Chemistry 286 20335-20344. (doi:10.1074/jbc. M110.215632)

Luo X \& Kraus WL 2012 On PAR with PARP: cellular stress signaling through poly(ADP-ribose) and PARP-1. Genes and Development 26 417-432. (doi:10.1101/gad.183509.111)

Matsuda F, Inoue N, Manabe N \& Ohkura S 2012 Follicular growth and atresia in mammalian ovaries: regulation by survival and death of granulosa cells. Journal of Reproduction and Development 58 44-50. (doi:10.1262/jrd.2011-012)

Megnin-Chanet F, Bollet MA \& Hall J 2010 Targeting poly(ADP-ribose) polymerase activity for cancer therapy. Cellular and Molecular Life Sciences 67 3649-3662. (doi:10.1007/s00018-010-0490-8)

Munoz-Gamez JA, Rodriguez-Vargas JM, Quiles-Perez R, AguilarQuesada R, Martin-Oliva D, de Murcia G, Menissier de Murcia J, Almendros A, Ruiz de Almodovar M \& Oliver FJ 2009 PARP-1 is involved in autophagy induced by DNA damage. Autophagy 5 61-74. (doi:10.4161/auto.5.1.7272)

Nguewa PA, Fuertes MA, Alonso C \& Perez JM 2003 Pharmacological modulation of poly(ADP-ribose) polymerase-mediated cell death: exploitation in cancer chemotherapy. Molecular Pharmacology 64 1007-1014. (doi:10.1124/mol.64.5.1007) 
Petrilli V, Herceg Z, Hassa PO, Patel NS, Di Paola R, Cortes U, Dugo L, Filipe HM, Thiemermann C, Hottiger MO et al. 2004 Noncleavable poly(ADP-ribose) polymerase-1 regulates the inflammation response in mice. Journal of Clinical Investigation 114 1072-1081. (doi:10.1172/ JCl200421854)

Qian H, Xu J, Lalioti MD, Gulle K \& Sakkas D 2010 Oocyte numbers in the mouse increase after treatment with 5 -aminoisoquinolinone: a potent inhibitor of poly(ADP-ribosyl)ation. Biology of Reproduction $\mathbf{8 2}$ 1000-1007. (doi:10.1095/biolreprod.109.080697)

Smulson ME, Pang D, Jung M, Dimtchev A, Chasovskikh S, Spoonde A, Simbulan-Rosenthal C, Rosenthal D, Yakovlev A \& Dritschilo A 1998 Irreversible binding of poly(ADP)ribose polymerase cleavage product to DNA ends revealed by atomic force microscopy: possible role in apoptosis. Cancer Research 58 3495-3498.

Spina-Purrello V, Patti D, Giuffrida-Stella AM \& Nicoletti VG 2008 Parp and cell death or protection in rat primary astroglial cell cultures under LPS/IFN $\gamma$ induced proinflammatory conditions. Neurochemical Research 33 2583-2592. (doi:10.1007/s11064-008-9835-1)

Tanori M, Mancuso M, Pasquali E, Leonardi S, Rebessi S, Di Majo V, Guilly MN, Giangaspero F, Covelli V, Pazzaglia S et al. 2008 PARP-1 cooperates with Ptc1 to suppress medulloblastoma and basal cell carcinoma. Carcinogenesis 29 1911-1919. (doi:10.1093/carcin/bgn174)

Tingen C, Kim A \& Woodruff TK 2009a The primordial pool of follicles and nest breakdown in mammalian ovaries. Molecular Human Reproduction 15 795-803. (doi:10.1093/molehr/gap073)

Tingen CM, Bristol-Gould SK, Kiesewetter SE, Wellington JT, Shea L \& Woodruff TK $2009 \mathrm{~b}$ Prepubertal primordial follicle loss in mice is not due to classical apoptotic pathways. Biology of Reproduction 81 16-25. (doi:10.1095/biolreprod.108.074898)
Virag L \& Szabo C 2002 The therapeutic potential of poly(ADP-ribose) polymerase inhibitors. Pharmacological Reviews 54 375-429. (doi:10. 1124/pr.54.3.375)

Walsh CM \& Edinger AL 2010 The complex interplay between autophagy, apoptosis, and necrotic signals promotes T-cell homeostasis. Immunological Reviews 236 95-109. (doi:10.1111/j.1600-065X.2010.00919.x)

Wei Q \& Shi F 2012 Cleavage of poly (ADP-ribose) polymerase-1 is involved in the process of porcine ovarian follicular atresia. Animal Reproduction Science 138 282-291. (doi:10.1016/j.anireprosci.2013. 02.025)

Wei Q, Shi F, He J, Xie C, Xu K, Zhang W, Sun S, Fedail J, Watanabe G \& Taya K 2012 Effects of exogenous $17 \beta$-estradiol on follicular development in the neonatal and immature mouse in vivo. Reproductive Medicine and Biology 11 135-141. (doi:10.1007/s12522-012-0122-0)

Wen X, Li D, Tozer AJ, Docherty SM \& Iles RK 2010 Estradiol, progesterone, testosterone profiles in human follicular fluid and cultured granulosa cells from luteinized pre-ovulatory follicles. Reproductive Biology and Endocrinology 8 117. (doi:10.1186/1477-7827-8-117)

Yelamos J, Farres J, Llacuna L, Ampurdanes C \& Martin-Caballero J 2011 PARP-1 and PARP-2: new players in tumour development. American Journal of Cancer Research 1 328-346.

Received 23 April 2013

First decision 23 July 2013

Revised manuscript received 28 July 2013

Accepted 23 September 2013 